Noor Dina Hashim, MD

Mohd Razif Mohamad Yunus, MBBS, MS (ORL-HNS)

Asma Abdullah, MD, MS (ORL-HNS)

Marina Mat Baki, MD, MS (ORL-HNS)

Salina Husain, MD, MS (ORL-HNS)

Mazita Ami, MBChB, MS (ORL-HNS)

Department of Otorhinolaryngology

Universiti Kebangsaan Malaysia Medical Centre
Correspondence: Dr. Mohd Razif Mohamad Yunus

Department of Otorhinolaryngology Universiti Kebangsaan Malaysia Medical Centre

Jalan Yaacob Latiff, Bandar Tun Razak

56000 Cheras, Kuala Lumpur, Malaysia

Phone: +60391455555 ext 6845

Fax: +60391737840

Email:razif72@gmail.com

The authors declared that this represents original material that is not being considered for publication or has not been published or accepted for publication elsewhere, in full or in part, in print or electronic media; that the manuscript has been read and approved by all the authors, that the requirements for authorship have been met by each author, and that each author believes that the manuscript represents honest work;

Disclosures: The authors signed disclosures that there are no financial or other (including personal) relationships, intellectual passion, political or religious beliefs, and institutional affiliations that might lead to a conflict of interest.

\section{Primary Malignant Parotid Lymphoma: Two Case Reports with Different Disease Progression and Treatment}

\begin{abstract}
Objective: To describe our experience in managing two cases of primary malignant parotid lymphoma.
\end{abstract}

\section{Methods:}

Design: Case Report

Setting: Tertiary University Referral Center

Patients: Two

Results: Both patients underwent superficial parotidectomy. Despite recurrence in one, the disease was controlled with conservative management. However, the disease was more aggressive in the other, requiring additional chemo-radiotherapy.

Conclusion: Malignant parotid lymphoma may present with varying stages, grades and clinical courses, requiring different management approaches. The treatment options are based on grading and staging at diagnosis and should be implemented depending on individual case.

Keywords: Parotid neoplasms, malignant lymphoma

The head and neck is the second most common site for extranodal lymphoma after the gastrointestinal tract. ${ }^{1}$ Usually it affects Waldeyer's ring, the nose, the paranasal sinuses and thyroid in descending order of frequency. Primary malignant lymphoma is only rarely found in the parotid gland and is mostly of the Non-Hodgkin B cell type. This type has various subtypes with differences in histology, behaviour, treatment and prognosis. We report two cases of primary malignant parotid lymphoma with different clinical courses and treatments.

\section{CASE REPORTS}

\section{Case 1}

A 74-year-old elderly male with diabetes, hypertension and bronchial asthma presented with a 7-year history of a painless, slow growing mass over the right angle of the mandible. He denied any associated constitutional symptoms. On examination, there was a $3 \times 3 \mathrm{~cm}$ firm, mobile non-tender single lymph node palpable over the right angle of the mandible. A Computed 
Tomography (CT) scan of neck showed a well-defined enhancing lesion measuring $2.4 \times 2.5 \mathrm{~cm}$, located in the superficial lobe of the right parotid gland with multiple subcentimeter lymph nodes seen in the posterior cervical triangle bilaterally. He underwent superficial parotidectomy, yielding a histopathological diagnosis of malignant parotid follicular Non Hodgkin Lymphoma Stage I. He was referred to the haematology service and a bone marrow biopsy showed no infiltration of lymphoma cells. In view of localized, early disease and his medical background, watchful observation was recommended.

The patient remained well on a regular follow-up until two and a half years later, when he presented with right submandibular and posterior cervical lymphadenopathy. An excision lymph node biopsy showed no malignant cells. However in about six months, the ipsilateral cervical lymphadenopathy had grown bigger and inguinal lymph nodes had also become palpable. Excision biopsies from both sites showed recurrent follicular lymphoma. A thorough CT scan showed multiple cervical, axillary, mediastinal and inguinal lymphadenopathies, but no solid organ involvement. There were no distant metastases. After another year, he again presented with right submandibular lymphadenopathy that was confirmed positive for recurrent disease. Subsequent CT scan showed similar findings to the previous scan but the lymph node sizes were reduced. The patient has otherwise been well throughout the years despite recurrent disease. The haematological team recommended supportive, palliative treatment in view of his age and multiple medical illnesses.

\section{Case 2}

A 14-year-old girl presented with a painless right pre-auricular swelling which gradually increased in size over six months. She also complained of loss of appetite with significant weight loss. There were intermittent episodes of fever but no night sweats. Physical examination revealed a firm, mobile, non-tender mass in the right parotid region measuring about $4 \times 4 \mathrm{~cm}$. It seemed unattached to overlying skin or underlying structures, and there was no palpable cervical lymphadenopathy. A fine-needle aspiration cytology examination was suggestive of pleomorphic adenoma. She underwent right superficial parotidectomy with a histopathologic diagnosis of Diffuse Large B Cell Non Hodgkin Lymphoma. She was referred to the haematology team and bone marrow aspiration showed no infiltration of lymphoma cells. However, she defaulted soon after.

She presented again six months later with bilateral lower limb weakness and back pain. A CT scan showed a residual mass in the right parotid region with bilateral subcentimeter cervical lymphadenopathies from levels I to V. Enlarged matted anterior mediastinal lymph nodes were also seen. There were no other solid organ metastases. Also noted were multiple lytic lesions in the spine, iliac, ischial and femoral bones. Further Magnetic Resonance Imaging (MRI) of the spine showed multiple lesions at various levels with collapse of T4 vertebrae and extension into the spinal canal. The patient had five courses of radiotherapy of $20 \mathrm{~Gy}$ to her spine followed by six courses of Bleomycin-FluouracilMethotrexate - 90 (BFM -90) regimen chemotherapy. During her first course, a whole body CT scan showed new lesions in both breasts and kidneys with worsening bony lesions. Fortunately, after completion of chemotherapy, the Positron Emission Tomography (PET) scan showed that she had responded well and was in remission. Post treatment MRI showed improvement of both bony and paraspinal lesions.

\section{DISCUSSION}

The incidence of primary malignant parotid lymphoma is very rare, accounting for $3-5 \%$ of all parotid tumours. ${ }^{1,2}$ The parotid gland is the commonest salivary gland to be affected by lymphoma, constituting about $91 \%$ of all salivary gland lymphoma as reported by Hyman et al. ${ }^{3}$ This is possibly due to the presence of lymph nodes and lymph aggregates in the parotid glands which are not found in the submandibular and sublingual glands. Parotid lymphoma may arise from intra-glandular lymph nodes or from the gland parenchyma. Parenchymal origin lymphoma is reported to be low grade, localized and often curable.

Malignant lymphoma of the parotid gland commonly occurs in 50 to 80 -year-olds. Sex predilection varies in many studies. According to Barnes et al. ${ }^{8}$ parotid lymphoma may be suspected in patients with a parotid mass and: history of malignant lymphoma; or with autoimmune disorders; or a previous diagnosis of benign lymphoepithelial lesion; or with multiple cervical lymphadenopathy; or in patients with multiple unilateral or bilateral parotid masses.

It is difficult to identify parotid lymphoma from the clinical history due to its limited value. According to Tiplady et al. ${ }^{4}$ only $12 \%$ of patients had systemic ' $B$ ' symptoms. B -symptoms are a group of symptoms that may be present in individuals with lymphoma which are important in identifying how the cancer is likely to behave. They include fever for three consecutive days, weight loss exceeding $10 \%$ of body weight within six months and drenching night sweats. Patients with B-symptoms have a little less chance of doing well after treatment. They are more significant in those with extensive disease such as our second patient. A history of autoimmune disease such as Rheumatoid Arthritis or Sjögrens' might be helpful as up to $44 \%$ may have coexistant parotid lymphoma. ${ }^{5}$

Clinically, malignant parotid lymphoma is almost indistinguishable from benign neoplasms. The commonest presentation is painless, 
progressively enlarging parotid mass. Twenty percent of cases are associated with cervical lymphadenopathy ${ }^{6}$ which is relatively rarely seen in benign parotid tumour and only $2 \%$ in parotid carcinoma. Other unusual signs and symptoms at presentation, as described by Naoki et al.' include pain (9\%), facial nerve paresis (4-15\%) and fixation of mass to overlying skin or underlying structures.

Hymann and Wolff ${ }^{9}$ - proposed three criteria to suggest primary lymphoma of the salivary gland; namely involvement of a salivary gland as the first symptom, histological evidence of lymphoma of the salivary gland parenchyma and presence of architectural and cytological confirmation of malignant nature of the lymphoid infiltrate. A confirmed diagnosis of malignant parotid lymphoma is often made after superficial or total parotidectomy. According to Tiplady et al. ${ }^{4}$ there is no clinical indication for total parotidectomy in parotid lymphoma. Partial removal of the gland is recommended as a lower incidence of facial nerve palsy is reported. The author has also pointed out their concern regarding facial cosmesis following total parotidectomy.

According to Wakely, ${ }^{5}$ Fine Needle Aspiration Cytology (FNAC) is a highly specific diagnostic test with $100 \%$ specificity and more than $80 \%$ sensitivity due to the use of immunophenotyping and cytogenetic techniques. Following a diagnosis by FNAC, a full staging of disease can be done, avoiding the risk of facial nerve palsy from total parotidectomy. If FNAC is non-diagnostic, it is safer to perform partial parotidectomy. However, FNAC may still fail to detect the actual diagnosis as seen in our second case.

The treatment and prognosis of malignant parotid lymphoma is based on the histological grading and staging at the time of diagnosis. The Ann-Arbor classification is used for staging and Working Fomulation (WF) classification is used for histological grading. Generally, stages I and II (localized disease) are treated with postoperative radiation, while multifocal or systemic disease is treated with chemotherapy. ${ }^{8}$

A study by Tiplady et $a l .^{4}$ reported various treatment regimens. Those with high grade disease received combination chemotherapy with Cyclophosphomide-Hydroxydoxorubicin-Oncovin-Prednisolone (CHOP) or BFM regimes. Patients with low grade lymphoma such as follicular lymphoma, were usually treated with chlorambucil regimens. Some underwent parotidectomy alone, others had radiotherapy alone or in combination with radiotherapy.

Sun et al. $^{6}$ reported BFM-90 regimen can greatly improve the survival rate of children and adolescents with B-Non Hodgkin Lymphoma (NHL), especially of patients with advanced NHL. CHOP or combined CHOP regimens work better for early stage patients, but produce low survival rate for patients with advanced NHL. It is suggested that treatment is given accordingly based on individual staging and grading. Case 1 showed the disease may remain quiescent after parotidectomy alone, while in the more advanced Case 2, the patient required chemotherapy and local radiotherapy for spinal metastases before achieving remission of the disease.

Overall survival rate can be predicted from histological grading. The 5 -year survival rate for low grade disease is $59 \%$, while it is $37 \%$ for high grade disease. ${ }^{3}$ Staging was not found to be statistically significant in determining prognosis in high grade disease but is significant in low grade disease ${ }^{8}$ where the 5 -year survival rate for Stage 1 disease is $69 \%$ compared with $44 \%$ for higher stages.

The prognosis of lymphoma presenting as a parotid mass is favourable especially in low grade disease. Therefore, early diagnosis and treatment of malignant parotid lymphoma may ensure an excellent survival.

\section{REFERENCES}

1. Watkin GT, MacLennan KA, Hobsley M. Lymphomas presenting as lumps in the parotid region. Br J Surg. 1984 Sep;71(9):701-702.

2. Mehle ME, Kraus DH, Wood BG, Tubbs R, Tucker HM, Lavertu P. Lymphoma of the parotid gland. Laryngoscope. 1993 Jan;103(1 pt 1):17-21.

3. Hyman GA, Wolff M. Malignant lymphomas of salivary glands. Review of the literature and report of 33 new cases, including four cases associated with lymphoepithelial lesion. Am J Clin Pathol. 1976 Apr;65(4):421-438.

4. Tiplady CW, Taylor PR, White J, Arullendran P, Proctor SJ; Scotland and Newcastle Lymphoma Group. Lymphoma presenting as a parotid tumour: a population-based study of diagnosis, treatment and outcome on behalf of the Scotland and Newcastle Lymphoma Group. Clin Oncol (R Coll Radiol) 2004 Sep;16(6):414-419.

5. Wakely P E Jr. Fine-needle aspiration cytopathology in diagnosis and management of malignant lymphoma: accurate and reliable? Diagn Cytopathol. 2000 Feb;22(2):120-125.

6. Sun XF, Su YS, Liu DG, Jiang WQ, He YJ, Lin TY, et al. Comparing CHOP, CHOP+HD-MTX, and BFM-90 regimens in the survival rate of children and adolescents with $B$ cell non-Hodgkin's lymphoma. Ai Zheng, 2004Aug;23(8):933-938.

7. Hirokawa N, Hareyama M, Akiba H, Satoh M, Oouchi A, Tamakawa M, et al. Diagnosis and treatment of malignant lymphoma of the parotid gland. Jpn J Clin Oncol 1998 Apr;28(4):245249.

8. Barnes L, Myers EN, Prokopakis EP. Primary malignant lymphoma of the parotid gland. Arch Otolaryngol Head Neck Surg. 1998 May;124(5):573-577.

9. Vega F, Lin P, Medeiros LJ. Extranodal lymphomas of the head and neck. Ann Diagn Pathol. 2005 Dec;9(6):340-350

10. Fujimura K, Yoshida M, Sugimoto T, Kuroda Y, Fujiyoshi T. Two cases of non-Hodgkin's lymphoma in the accessory parotid gland. Auris Nasus Larynx. 2004 Jun; 31(2):195-198. 